\title{
FAULT NODE RECOVERY ALGORITHM FOR ENHANCINGTHE LIFETIME OF AWIRELESS SENSOR NETWORK
}

\author{
S. Dawood \\ S. Abuelenin \\ A. Atwan \\ Faculty of computers and Information, Mansoura University, Egyp \\ saad.deved40@gmail.com \\ dr.sherihan@yahoo.com \\ atwan_2@yahoo.com
}

\begin{abstract}
Wireless Sensor Network (WSN) is type of network which consists of collection of tiny device called sensors nodes. In real wireless sensor networks, the sensor nodes use battery power supplies and thus have limited energy resources. This paper decrease the number of fault node and loss data and number of routing in the network based on enhanced Grade Diffusion with using Shortest Best Path. In the simulation, number of hop, power consumption, fault detection accuracy and time number of neighbour nodes measure the proposed algorithm. The proposed algorithm is also compared with distributed fault detection (DFD) and fault node recovery (FNR).
\end{abstract}

Keywords: WSNs, failure detection, failure recovery algorithm, Grade Diffusion, and dead node Shortest Save Path, and Fault Node Recovery.

\section{Introduction}

Wireless Sensor Network is a sort of system which contains of collection of very small devices called sensors nodes transferring data between them. The end user/observer can access the data [1]. Due to small size and low cost and limited energy resources and it is not possible to recharge them [2].So energy is a main constraint in WSN operation. Sensor nodes are small, which are capable of local processing, low-power, distributed devices, inexpensive [3].

Each sensor node is capable of only a limited amount of processing. , they have the ability to measure a Small physical environment in great detail. A sensor node it works to integrate the abilities to compute sense and communicate the all features inside the single chip. The structure of sensor node is the battery is the primary energy supply of node of the sensor. Then there exists central processing unit or micro processor which performs all processing activities. The processor is attached with a small memory unit which is used to store data [4]. All the nodes work with some to send and receive the required information. In addition, each sensor node is typically equipped with a radio transceiver or other wireless communications device or antenna which is used to communicate with other neighbour nodes. Wireless Sensor Network (WSN) contains hundreds or thousands of sensor nodes, each sensor able for communication and computation and senses data or gather information about the events occurring near that sensor, then the sensor nodes process those data gathered and at last those data are transmitted to other sensor nodes or base stations and also a sensor node can receive data from neighbour nodes. This way the sensor nodes create a network [5]. 


\section{Related works}

We briefly review the related works in WSNs of fault detection and recovery. Anuradha M S, DeepaPatil [6] suggested a filer node recuperation algorithm to improve that age of WSN when few nodes dead. The algorithm is predicated on the fault node route revelation and fault node detection algorithm.

Elmira Moghaddami Khalilzad et al. [7] proposed an incipient recovery algorithm predicated on inheritor cull. Antecedent algorithms do bunch headcull when every shortcoming happens but the suggested algorithm does this cull once and can cull group head quickly and without an extreme amount of estimation.

M. Merabti [8] utilized a heartbeat predicated testing mechanism to detect failure in each cluster and take the advantage of cluster predicated architecture to forward the failure report to other cluster and their respective members.

Ann T. Tai Kam S. Tso, [9] used a cluster-predicated communication design to sanction the (Failure Detection Accommodation) to be implemented in a dispersed way by means of intra-cluster heartbeat diffusion and sent massage clusters through the upper layer of the communication. They widely develop the message excess that is innate in ad hoc wireless settings to alleviate the possessions of message loss on the precision and plan property of failure discovery.

HoJiun -Huei et al [10] Proposed LDA(Ladder diffusion algorithm) that is less power of time the path for data transmission in a wireless sensor network, utilizing less power and time to build. This algorithm is utilized for load balancing, in case in transmission efficiency and life of the sensor node due to redundant relay. The LDA evade power consumption.

\section{Proposed system}

A system has been developed for fault node recovery algorithm for WSNs based on Grade Diffusion algorithm combined with a new method called 'Saved Shortest Path'. The Enhanced Grade Algorithm is built to work on deploying nodes then choosing the shortest path after saving it inside the functioning node to identify the breakdown node. Choosing the shortest path is based on the current node power against predefined threshold by the sender for all nodes then determining the amount of power required to perform this operation. After choosing the shortest path, if the power inside the node is enough, it should be selected. If the power inside the node is not enough, it will be isolated and ignored as dead node which will be replaced by the following node, then to reconstruct the entire network as it deals with the system in case there are several breakdown node. The saved information for the Shortest Path deals with the network as a whole. The algorithm set forth in this paper depends on the GD algorithm, with the objective of replacing fewer sensor nodes that are inoperative or have exhausted batteries, and by reusing the greatest number of routing paths. These enhancements will be lower upgrade the WSN lifetime and reduce sensor node replacement cost. The grade diffusion algorithm and 'Saved Shortest Path' depend on the greater value of power of all node powers. The proposed algorithm introduces less replacement of sensor nodes and more reused routing paths. The proposed algorithm used the number of active nodes with reducing the rate of data loss in addition to reducing the rate of energy consumption. The routing table information unit provides one receiving the unique ID code, the sensor node will broadcast a guide plan from time to time so as to inform other nearby nodes of the existence of this simulation node. Specific Route determination is the process of maintaining a neighbor table for neighboring nodes and randomly selects a node with a lower path as the "fault node." Then the power consumption evaluation 
system node will insert its unique ID code into the header of the packet and submit the packet to the relay node. After receiving this packet, the relay nodes whose main task is communication with additional sensor will randomly select a node, which is closest to the management unit, from its routing table, and send the packet to the closest node. Thereafter, through Zero or multiple relay nodes, the packet will be forwarded to the management unit. The system of proposing GD is transmitted to node routing method. and the down link packet routing method. Permanently, the proposed will re-pack the packet With a destination's ID code and some routing information originally classified in the management unit and sent the packet out. Through multiple relay nodes, the GD will be recovering the failure node from the nearest node to the destination ID code. In the proposed algorithm, the depth $d(v)$ of node $v$ as the shortest distance of a node $\mathrm{v}$ from sink in $\mathrm{T}$, expressly, the least number of hops mandatory for a reading to get to sink from $\mathrm{v}$.

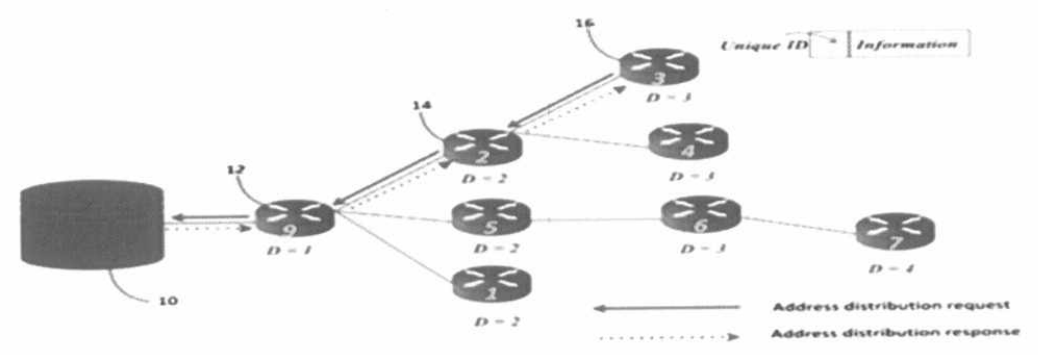

Figure (1) method of routing nodes in the proposed algorithm

Figure 1 is the method of routing and distributing the unique ID codes to the nodes depending on a head node. The virtual wireless device in this system includes a management unit 10, a gateway 12 , and numerous nodes 14. Wherein No.3 node's parent node is No. 2 node, and defines the No. 3 node a number 16. Each node includes a "depth" that illustrates the depth $d(v)$ of node $v$ as the shortest distance of a node $\mathrm{v}$ from sink in $\mathrm{T}$, expressly, the least number of hops mandatory for a reading to get to sink from $\mathrm{v}$. Therefore, indicating the distance between the node 14 and the management unit 10 . Since the method of detecting nearby nodes and maintaining the neighbor table is considered well known in the appropriate and additional details are therefore excluded for the shortest path length. The management unit 10 provides one unique ID code for each node. For example, as shown in Figure 1, the unique ID code for the node 16 is indicated (3). After receiving this unique ID code, the node will broadcast a guide frame repeatedly to inform other neighboring nodes of the entity of the node.

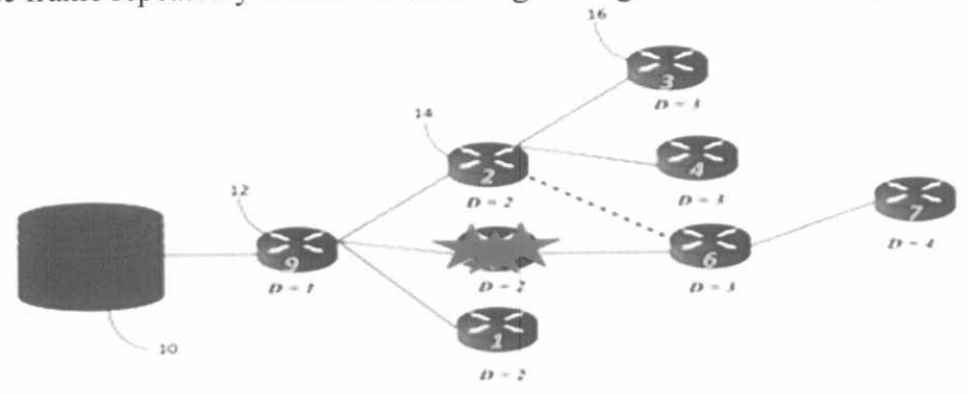

Figure (2) The low power consumption of one node in the proposed algorithm.

Figure 2 is an example of the low power consumption of one node that is faulty in the routing path of the present simulation. Management unit 10 and the head node (5) is fault, the node (6) choose 
randomly select other node (namely the node (1) or (2)) neighboring to the management unit 10 as the "next hop" to node on the source graph to gain the shortest route to the source management unit route the packet. In this paradigm, the node (6) selects the node (2) as the next hop. This paper aims algorithm that combined the shortest paths and Grade Diffusion as shown in Figure 3. Therefore, finding the confident quantity of paths betwixt a pair of source target nodes is used Enhanced GD. This is location on a faulty node. First, allow displaying the source node by $\mathrm{S} \in \mathrm{M}$ form that is demanded to obtain the sink node $t \in M$, consequently $\mathrm{K}$ shortest loop-less paths. These paths from a set of $\mathrm{K}$ constituents, $\mathrm{P}=\{\mathrm{P} 1, \mathrm{P} 2, \ldots, \mathrm{PK}\}$. Concurrently, every path of the latter set is defined by the sequence of $\mathrm{PK}=\langle\mathrm{s}=\mathrm{v} 1 \mathrm{~K}, \mathrm{v} 2 \mathrm{~K}, \ldots \ldots, \mathrm{viK}=\mathrm{t}>, \mathrm{n}=$ node transfer information where viKis the $\mathrm{i}$-th node of the $\mathrm{K}$ th shortest path.

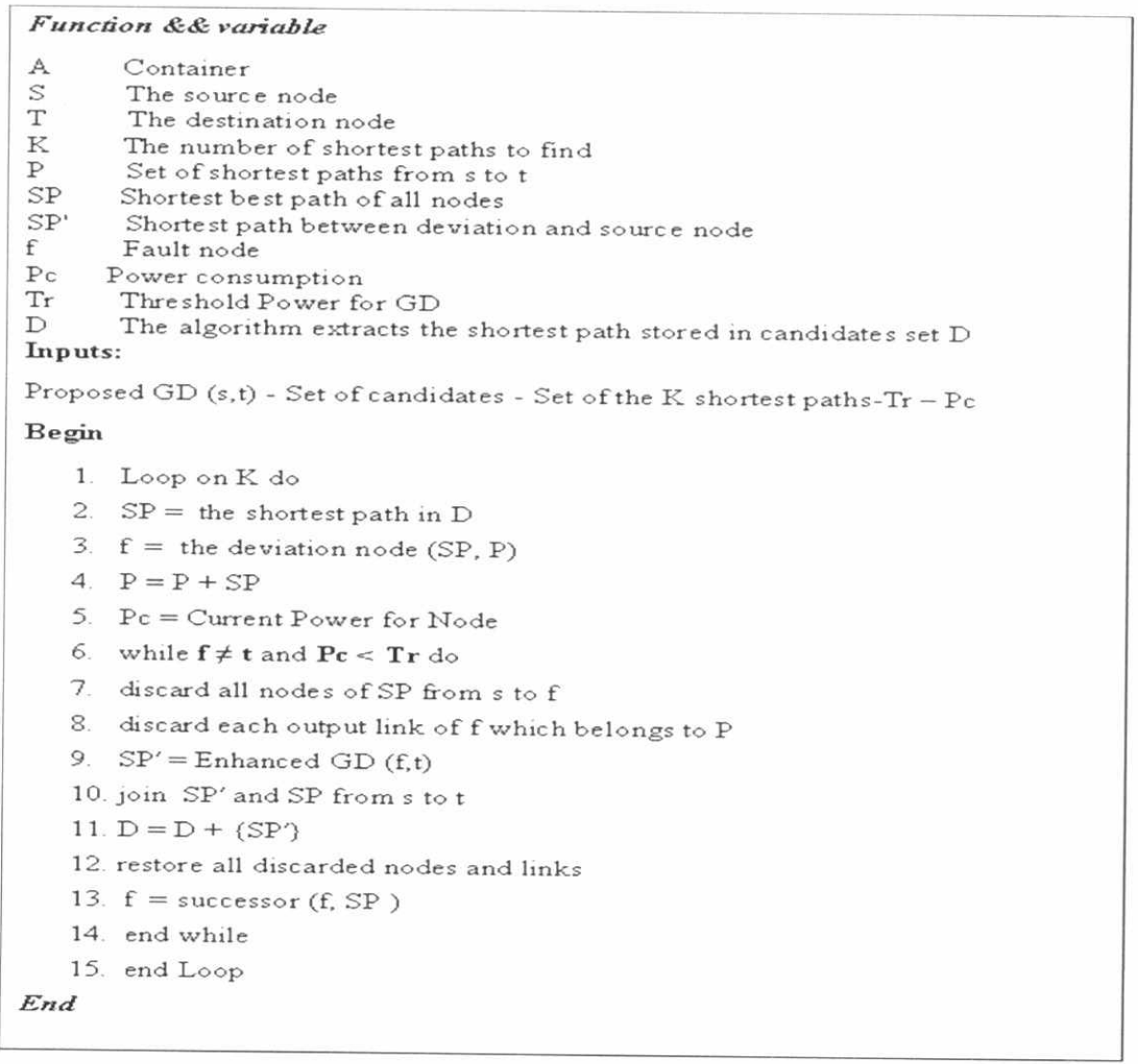

Figure(3) The Proposed Algorithm

WSNs are its number of node distributed where it is working cooperatively with the transfer of information between nodes based on choosing the shortest path. The algorithm is launched by calculating the shortest path P1 and launching some variables. Subsequently, the algorithm will perform $\mathrm{K}$ repetitions. In the process of being figured out from Algorithm, given an adjacency matrix and a source-sink pair of nodes. The algorithm will perform $\mathrm{K}$ repetitions. At the $\mathrm{k}$-th repetitions, the algorithm extracts the shortest path stored in candidates set D. The algorithm is calculated from all the 
$\mathrm{K}-1$ paths in S $\mathrm{P}$ by cause of escaping re-arithmetic of the previously computed paths. Some nodes do not work where they are replaced by a node depending choose the shortest route possible from source to sink. The algorithm reject confirmed nodes and links as it is presented. The shortest path SP 'between fault node and source node, the algorithm is initiated by calculating the shortest path P1 and internalizing some mistakes. Then the algorithm will perform $\mathrm{K}$ repetitions. At the $\mathrm{k}$-th repetition, the algorithm resumes the shortest path stored in candidates set $\mathrm{D}$. The algorithm is arithmetic with the remaining graph. Then, $\mathrm{S} \mathrm{P}^{\prime}$ joins the sub-path of the $\mathrm{K}$-th shortest path from source to fault node and saves this new developed path in the candidate set D. Finally, all the links and nodes beforehand rejected are repaired and the algorithm progress to the successor of fault node in the K-th shortest path. The system development executive is the platform that is located in the process of exposure in the Figure 3. Presentation systematization is development criteria the nodes are expanded narrow within the area limits.

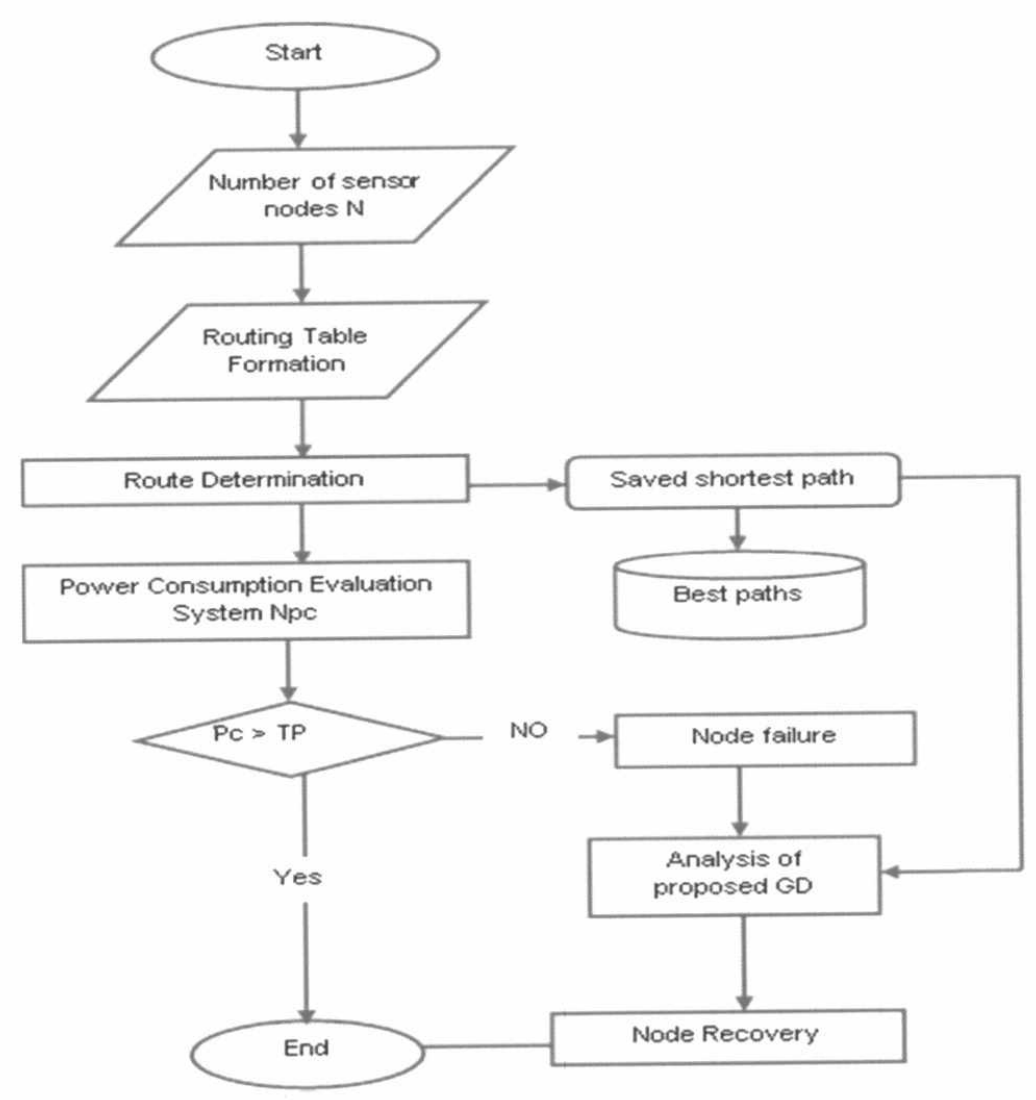

Figure (4)The flow chart of the system design

As shown in figure (4), the system design consists of Node Deployment Algorithm, is qualified to deploying the nodes in an appropriate location. The location of the nodes is in the proposed area. The sensor node executes deployment algorithm foundation on sensing range. Subsequently, the system gains input factors and use Routing Table Formation the algorithm is used to scheme to rout tables for 
all of the nodes. The routing table formation is output in each node and end-to-end path between node (u) and head (S)and use Route Determination This process consists of deciding which route from the sink node to destined node with the usage the control packets. Therefore, the route should be found in a way that the consumption of batteries inside the node is decreased throughout the network. It also aims to increase the lifetime and all saved lowest paths are backed up in database assuming that this route includes fault node and which best spare. That energy efficient routing determination is more critical than uncomplicated shortest path routing. We proposed new route detection method is chosen to decrease the power consumption. The number of outdated nodes will be compared to fault node recovery algorithm. The algorithm suggested will also decide the percentages of the amount of energy contained within each node around $40 \%$ or $70 \%$ and relying on these ratios to determine the ability of each node in the transmission of information. Where they were the amount of power to an existing inside node, better from threshold be selected; however, as the node has less than threshold will be considered as unsuccessful and should choose other node neighbouring them.

The Proposed GD algorithm not only creates the routing for each sensor node but also identifies a set of neighbour nodes to reduce the transmission loading the main two ideas are combined as below:

- $\quad$ Saved Shortest Path in Repository.

- $\quad$ Power check sum threshold.

The power usage of the sensor nodes in WSNs is inevitable. The system used proposes an algorithm to find and replace less sensor nodes and to reuse the least routing paths. Conventional search techniques are often unable to optimize nonlinear roles with various variables. One method, the system is a directed provides a power threshold as main reference of developed system, based on the concept of minimum level of power. This algorithm doesn't just reuses the most routing paths to enhance the WSN lifetime, but also decreases the replacement energy.

Every time scanning operation done, discovering all paths as alternatives and save the best short path in all neighbour nodes in shared repository called "Saved Shortest Path" that saved in head nodes only.

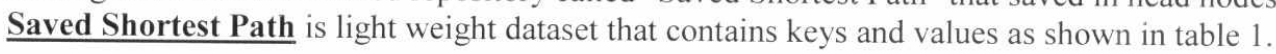

Table 1: calculation of best path

\begin{tabular}{|c|c|c|}
\hline Source & Destination & Best Path calculated from proposed GD \\
\hline Node $\mathrm{x}$ & Node $\mathrm{Y}$ & $\mathrm{X}-\mathrm{A}-\mathrm{Z}-\mathrm{G}-\mathrm{Y}$ \\
\hline Node $\mathrm{H}$ & Node $\mathrm{J}$ & $\mathrm{H}-\mathrm{E}-\mathrm{Q}-\mathrm{F}-\mathrm{X}-\mathrm{J}$ \\
\hline Node $\mathrm{Q}$ & Node $\mathrm{X}$ & $\mathrm{Q}-\mathrm{R}-\mathrm{P}-\mathrm{W}-\mathrm{C}-\mathrm{X}$ \\
\hline$\ldots$ & $\ldots$ & $\ldots$ \\
\hline Node $\mathrm{Z}$ & Node $\mathrm{K}$ & $\mathrm{Z}-\mathrm{Q}-\mathrm{A}-\mathrm{Q}-\mathrm{K}$ \\
\hline
\end{tabular}

All neighbour nodes contain this repository for resolving the issues of Grade Diffusion algorithm by sending out to the neighbouring nodes in the closest neighbouring set. Then, the nodes are chosen based on the hop-count. However, for resolving this issue, the next section is combined as "Power check sum threshold". The package-creating grade or the interested query packets must first be sent out. After that, the sensor nodes send the event information to the sink node, based on the proposed algorithm when fitting events happen.

In the proposed method, before transmitting the information, the saved pathway from each sensor to the sink node must be proactive. This could be achieved through a global way, with the lowest-cost, setup 
route starting from the sink nodes. Where that the head node depends on the schedule of information in determining the best path of using the existing data base to escape from calculations carried out by the repeated and to keep on head node of failure. After that, each node saves the data in its routing table.

Where paths are determined on the basis of cost:

- (The cost from the existing node to the sink node the length of the lowest- cost path).

- (The next hop from the existing node the length of the lowest- cost path).

The costs could be the hop number, energy consumption, delay, remaining energy, etc. The minimum level energy is use as the metric of power depends on the threshold it represents the amount of energy needed to perform the task. On the basis of the amount of the task and the required amount of energy contained in the node is to choose the appropriate path. In the example we use, there nodes in the networks shown in figure 2 .

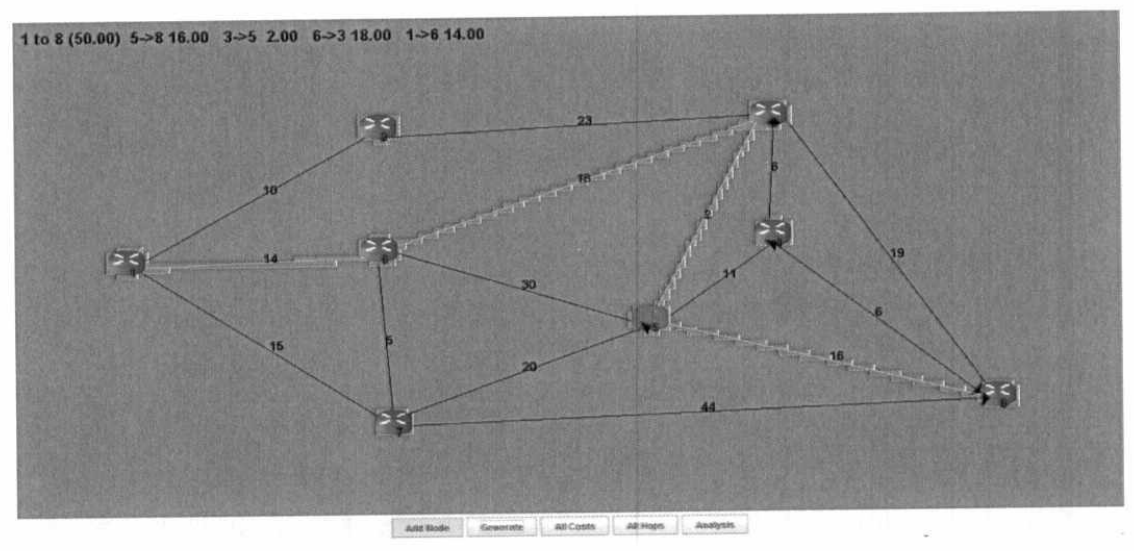

Figure (5) WSN without failure

To get the shortest-path from source node (1) to destination (8), we use Generate button which shows the shortest path from the source to the destination using proposed Grade Diffusion algorithm as shown in figure (5). From 1 to 8 , it will be unit $1 \rightarrow 6 \rightarrow 3 \rightarrow 5 \rightarrow 8$ As it imposes the shortest should be fastest way to transfer messages between two active nodes. 


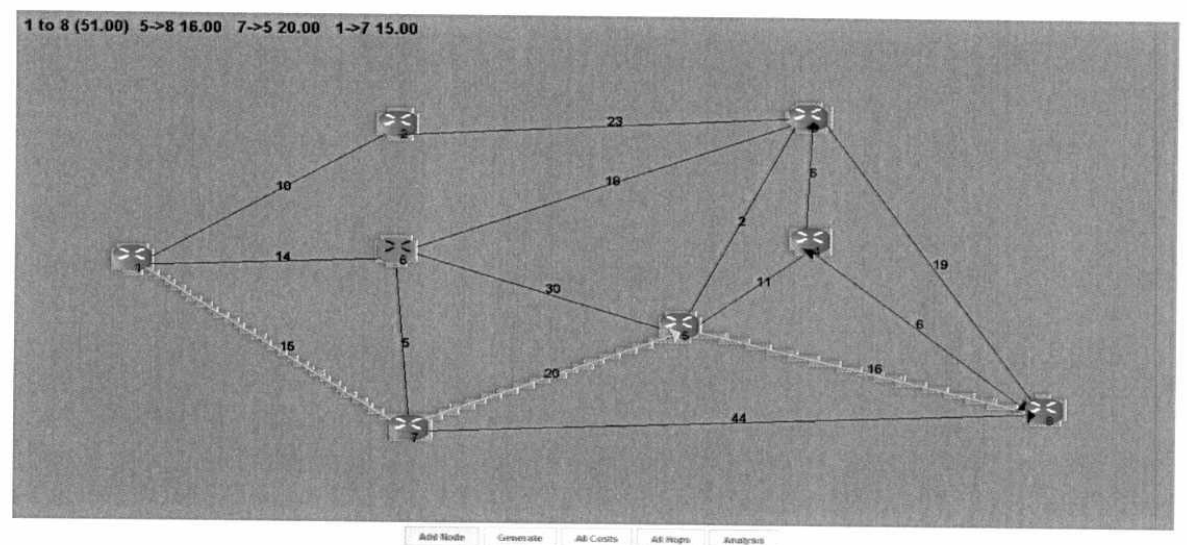

Figure (6) WSN with failure Recovery

In case of a failed node (6) as an example, the source node depends on data set to take shortest path; the path will be changed into $1 \rightarrow 7 \rightarrow 5 \rightarrow 8$ instead of $1 \rightarrow 6 \rightarrow 5 \rightarrow 3 \rightarrow 8$, Its now minimum level power which is the logical result. A failed node would result in a minimum level power as shown in figure 6 .

In this proposed system node failure is determined when they arrive to the extent critical identified before they fail and take the necessary requirements to identify alternative ways and thus is detected node failure in real time. It increases the life of the network and eliminates data loss.

The proposed Grade Diffusion selects the appropriate path depending on the amount of power located within each node, In the case of receiving request from an active node to the next node the power level becomes close of the lower threshold the source node starts to send a message to the data set to change the path and take best path.

In case of failure the system updating main "Saved Shortest Path Repository" by replacing the dead node by best alternative in the restructuring of the network, but not in general be divided into two failure network parts (before part) and (after part), This process will be determined fault node and isolated and find a replacement so that it can be linked to two parts with each other.

\section{Results}

The proposed algorithm based on saved shortest path is implemented a. Using schemes on the system's hardware can be expensive, thus, they should be proven to be accurate and feasible before using them. Therefore, simulation studies to be the best alternative way of testing, evaluating and verifying. The proposed algorithm scheme was implemented and compared with the FNR algorithm. The simulation tools accessibility available are Network Simulation 2 (ns-2), the Java Simulator (J-Sim) and SENSE. The various parameters such as calculating the number of hops and number of dead node and a live node are calculated and compared of fault node recovery algorithm (FNR). 
TABLEL2: ROUT MATRIX

\begin{tabular}{|c|c|c|c|c|c|c|c|c|c|c|c|c|c|c|c|c|c|c|c|c|c|c|}
\hline & 0 & 1 & 2 & 3 & 4 & 5 & 6 & 7 & 8 & 9 & 10 & 11 & 12 & 13 & 14 & 15 & 16 & 17 & 18 & 19 & 20 & 21 \\
\hline 0 & - & 0 & 0 & 0 & 0 & 0 & 0 & 1 & 1 & 1 & 3 & 10 & 2 & 2 & 10 & 14 & 14 & 16 & 15 & 8 & 19 & 20 \\
\hline 1 & 1 & - & 0 & 1 & 0 & 0 & 0 & 1 & 1 & 1 & 3 & 10 & 2 & 2 & 10 & 14 & 14 & 16 & 15 & 8 & 19 & 20 \\
\hline 2 & 2 & 0 & - & 2 & 0 & 0 & 0 & 1 & 1 & 3 & 3 & 12 & 2 & 2 & 10 & 14 & 14 & 16 & 15 & 9 & 19 & 20 \\
\hline 3 & 3 & 3 & 3 & - & 0 & 0 & 0 & 1 & 1 & 3 & 3 & 10 & 2 & 2 & 10 & 14 & 14 & 16 & 15 & 9 & 19 & 20 \\
\hline 4 & 4 & 0 & 0 & 0 & - & 4 & 0 & 9 & 1 & 1 & 3 & 10 & 2 & 2 & 10 & 14 & 14 & 16 & 15 & 8 & 19 & 20 \\
\hline 5 & 5 & 0 & 0 & 0 & 5 & - & 5 & 1 & 1 & 1 & 3 & 10 & 2 & 2 & 10 & 14 & 14 & 16 & 15 & 8 & 19 & 20 \\
\hline 6 & 6 & 0 & 0 & 0 & 0 & 6 & - & 1 & 9 & 1 & 3 & 10 & 2 & 2 & 10 & 14 & 14 & 16 & 15 & 8 & 19 & 20 \\
\hline 7 & 1 & 7 & 0 & 1 & 0 & 0 & 0 & - & 7 & 1 & 3 & 10 & 2 & 2 & 10 & 14 & 14 & 16 & 15 & 8 & 19 & 20 \\
\hline 8 & 1 & 8 & 0 & 1 & 0 & 0 & 0 & 8 & - & 8 & 9 & 10 & 2 & 2 & 10 & 14 & 14 & 16 & 15 & 8 & 19 & 20 \\
\hline 9 & 1 & 9 & 3 & 9 & 0 & 0 & 0 & 1 & 9 & - & 9 & 10 & 2 & 2 & 10 & 14 & 14 & 16 & 15 & 9 & 19 & 20 \\
\hline 10 & 3 & 3 & 3 & 10 & 0 & 0 & 0 & 1 & 9 & 10 & - & 10 & 11 & 2 & 10 & 14 & 14 & 16 & 15 & 9 & 19 & 20 \\
\hline 11 & 2 & 3 & 12 & 10 & 0 & 0 & 0 & 1 & 9 & 10 & 11 & - & 11 & 2 & 10 & 14 & 14 & 16 & 15 & 9 & 19 & 20 \\
\hline 12 & 2 & 0 & 12 & 2 & 0 & 0 & 0 & 1 & 1 & 10 & 11 & 12 & - & 2 & 10 & 14 & 14 & 16 & 15 & 9 & 19 & 20 \\
\hline 13 & 2 & 0 & 13 & 2 & 0 & 0 & 0 & 1 & 1 & 3 & 3 & 12 & 2 & - & 10 & 14 & 14 & 16 & 15 & 9 & 19 & 20 \\
\hline 14 & 3 & 3 & 3 & 10 & 0 & 0 & 0 & 1 & 9 & 10 & 14 & 10 & 11 & 2 & - & 14 & 14 & 16 & 15 & 9 & 19 & 20 \\
\hline 15 & 3 & 3 & 2 & 10 & 0 & 0 & 0 & 1 & 9 & 10 & 14 & 10 & 11 & 2 & 15 & - & 14 & 18 & 15 & 9 & 19 & 20 \\
\hline 16 & 3 & 3 & 2 & 10 & 0 & 0 & 0 & 1 & 9 & 10 & 14 & 10 & 11 & 2 & 16 & 14 & - & 16 & 16 & 9 & 19 & 20 \\
\hline 17 & 3 & 3 & 2 & 10 & 0 & 0 & 0 & 1 & 9 & 10 & 14 & 10 & 11 & 2 & 16 & 18 & 17 & - & 17 & 9 & 19 & 20 \\
\hline 18 & 3 & 3 & 2 & 10 & 0 & 0 & 0 & 1 & 9 & 10 & 14 & 10 & 11 & 2 & 16 & 18 & 18 & 18 & - & 9 & 19 & 20 \\
\hline 19 & 1 & 8 & 3 & 9 & 0 & 0 & 0 & 8 & 19 & 19 & 9 & 10 & 2 & 2 & 10 & 14 & 14 & 16 & 15 & - & 19 & 20 \\
\hline 20 & 1 & 8 & 3 & 9 & 0 & 0 & 0 & 8 & 19 & 19 & 9 & 10 & 2 & 2 & 10 & 14 & 14 & 16 & 15 & 20 & - & 20 \\
\hline 27 & 1 & 8 & 3 & 9 & 0 & 0 & 0 & 8 & 19 & 19 & 9 & 10 & 2 & 2 & 10 & 14 & 14 & 16 & 15 & 20 & 21 & - \\
\hline
\end{tabular}

The simulation program testes on both systems by running all cases as shown in table 2. As 20 X 20 nodes all paths displayed in this matrix is executed just like each path for all possibilities.

For instance;

$1-2$

$1-2-3$

1-2-3-4

$1-2-3-4-5 \ldots$ Etc As confirmed that GD works well with partial topological information.

In this paper, the proposed grade diffusion algorithm and fault node recovery algorithm are compared. Here the power consumption in mw is calculated and compared with this fault node recovery results. Hence, from the output result of comparison, the performance of proposed grade diffusion algorithm is much preferable than fault node recovery algorithm as you can see next steps. The power consumption in mw is measured by testing number of scale ns from 10 to 100 nodes. Then the proposed GD and FNR compared in fig 5. From the results, it is noticed proposed grade diffusion has less Power Consumption compared to FNR.

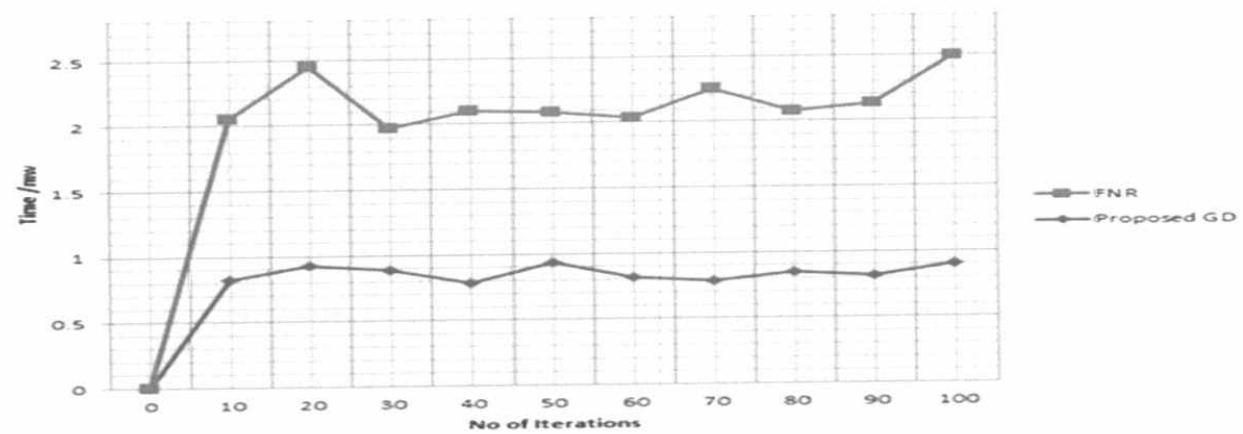

Figure (7) Power consumption of Fault Node Recovery algorithm and the proposed grade diffusion 
Different number of neighboring nodes and node failure of the fault-detecting accuracy of proposed GD and fault detection accuracy of DFD scheme are presented in Table.3. Each in order, from which that the fault detection accuracy of proposed GD rises with the lessening of the average number of neighboring nodes hold on to physically high fault detection accuracy rising of detection failure node. Fault detection accuracy of proposed GD method is certainly upward than DFD scheming. Aside from that proposed GD scheme maintains high fault detection accuracy even with upper node's failure and a small average number of neighbor nodes.

Table (3) Fault Detection Accuracy of Proposed GD and DFD

\begin{tabular}{|c|c|c|c|c|c|c|}
\hline \multirow{2}{*}{\multicolumn{2}{|c|}{$\begin{array}{l}\text { Average number } \\
\text { Of netghbor node }\end{array}$}} & \multicolumn{5}{|c|}{ Eatture Node } \\
\hline & & 0.1 & 0.15 & 02 & 0.25 & 03 \\
\hline \multirow{2}{*}{5} & $\begin{array}{l}\text { Proposed } \\
\text { GD }\end{array}$ & $:$ & $:$ & : & 0.98 & $0.9^{-}$ \\
\hline & $D=D$ & 0.998 & 0.985 & 0.962 & 0.935 & $0.5^{7} 3$ \\
\hline \multirow{2}{*}{10} & $\begin{array}{l}\text { Proposed } \\
\text { GD }\end{array}$ & $:$ & : & : & $0.9^{-}$ & 0.94 \\
\hline & DED & : & 0.992 & 0.976 & 0.955 & 0.917 \\
\hline \multirow{2}{*}{15} & $\begin{array}{l}\text { Proposed } \\
\text { GD }\end{array}$ & ! & : & : & 0.95 & 0.9 \\
\hline & $D E D$ & : & : & 0.993 & $0.98:$ & 0.968 \\
\hline \multirow{2}{*}{20} & $\begin{array}{l}\text { Proposed } \\
\text { GD }\end{array}$ & : & : & : & 0.92 & $0.5^{-}$ \\
\hline & D D & : & : & 0.993 & 0.98: & 0.968 \\
\hline
\end{tabular}

From figs. 8 to 9, it is noticed of entering different number of nodes diffused, the median number of neighbor nodes and the nodes failure, the proposed GD platform clearly out performs the DFD scheme. The fault detection accuracy of the DFD platform intently lessees following lessening of the node's failure. On the other hand, the proposed GD platform performs preferable than the DFD platform for node fault detection. 


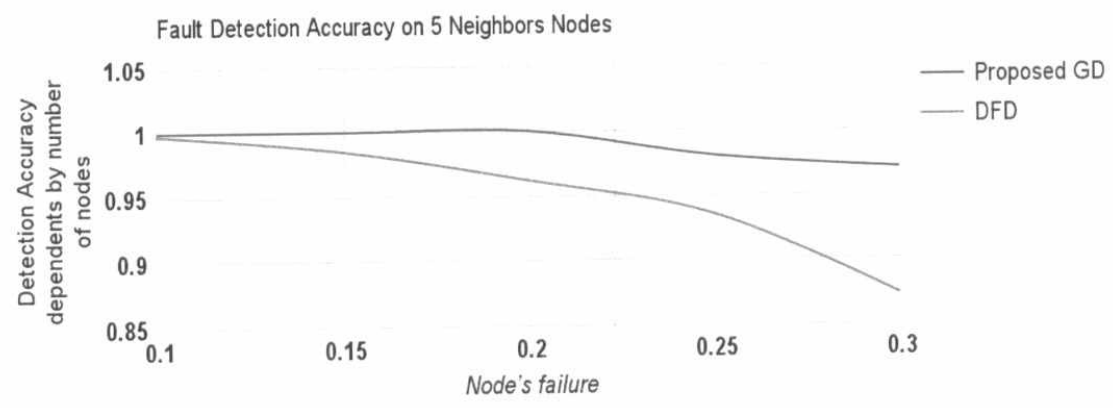

Figure (8) The accuracy of the detection for different node failures upon deploying 200 nodes at random and the median number of neighboring is 5 .

The calculated correctness trending different median numbers of neighboring nodes in figure (8) the failed node ratio is seen to be 0.3 . We can be see that the fault detection correctness of the Grade Diffusion proposed and Distributed Fault Detection method increases with the rise of average numbers of neighboring node sets. The tendency of faulty node detection correctness with different node failure ratios is also analyzed when 200 nodes are randomly deployed and the median numbers of neighbor nodes is 10 demonstrating that the fault detection correctness of the proposed Grade Diffusion and the Distributed Fault Detection scheme decreases with the increase in the node failure ratio and the proposed Grade Diffusion scheme also outperforms the Distributed Fault Detection scheme.

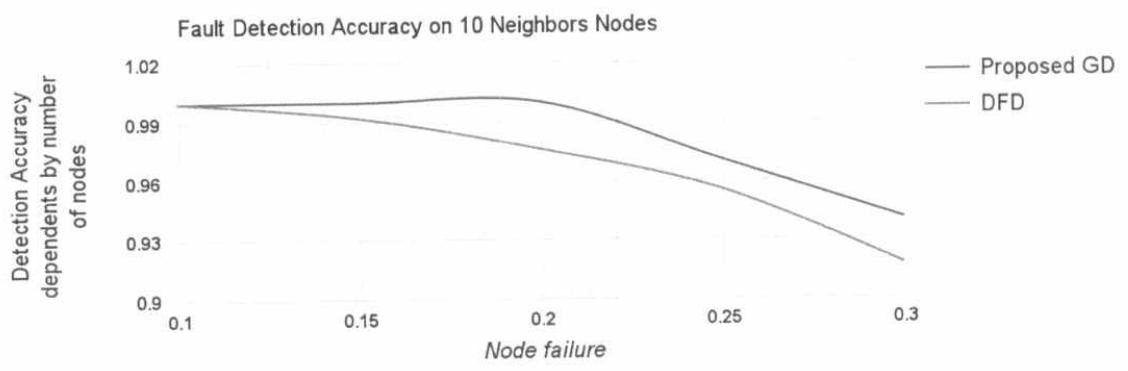

Figure (9) The accuracy of the detection for different node failures upon deploying 200 nodes at random and the median number of neighboring is 10 .

The number of neighboring D and nodes' failure ratio $\mathrm{b}$, from that the fault detection correctness of the two methods decreases with the declining of D and inclining of $b$. For the same D and b, the fault detection accuracy of Grade Diffusionproposed method is proven to bemore than Distributed Fault Detectionmethod. Asides from that, the proposed Grade Diffusionmethod can also make the fault detection accuracy remain highalong with the high node failure ratio and least number of neighboring nodes. The change of fault discovery efficiency of the two methods with node failure ratios for dissimilar average numbers of neighboring nodes are compared as shown in Figure 10 and 11. 


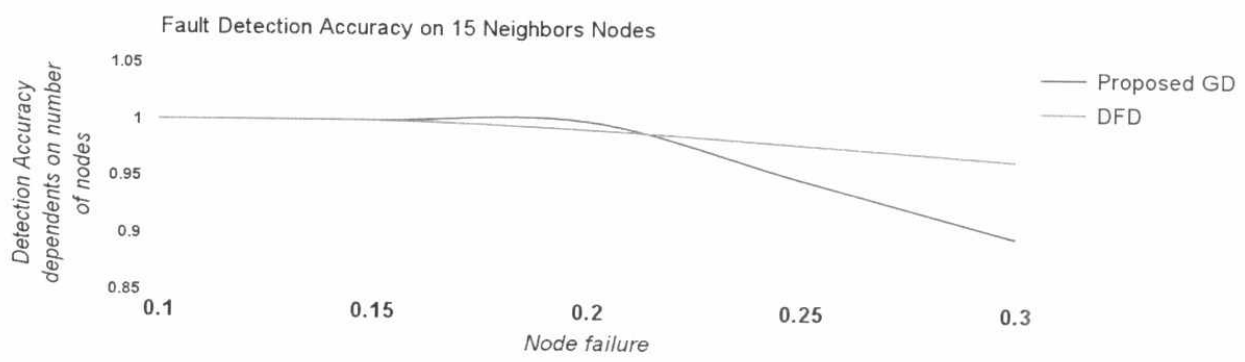

Figure (10) The accuracy of the detection for different node failures upon deploying 200 nodes at random and the median number of neighboring is 15 .

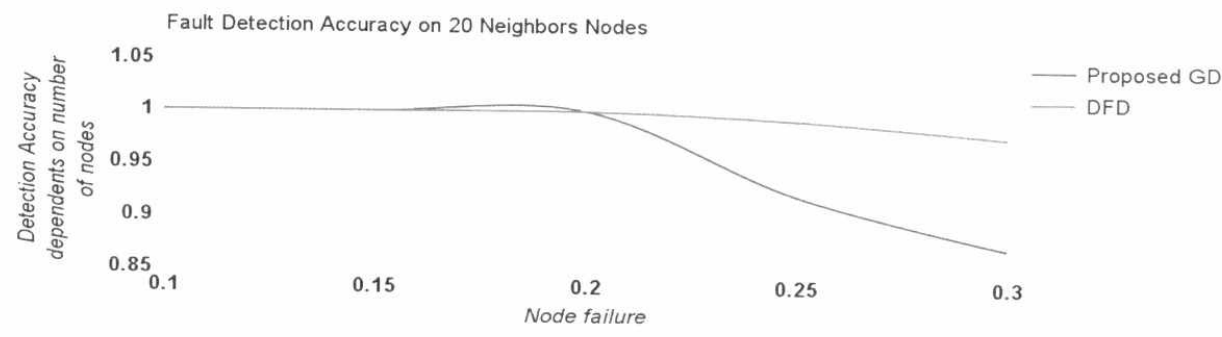

Figure (11) The accuracy of the detection for different node failures upon deploying 200 nodes at random and the average number of neighboring nodes is 20 .

As shown in Figure12. The number of hops for the routes is discovered using Proposed Grade Diffusion and Fault Node. In this results we can see prove Proposed Grade Diffusion has less number of hops as compared of Fault Node then both systems are tested in same circumstances.

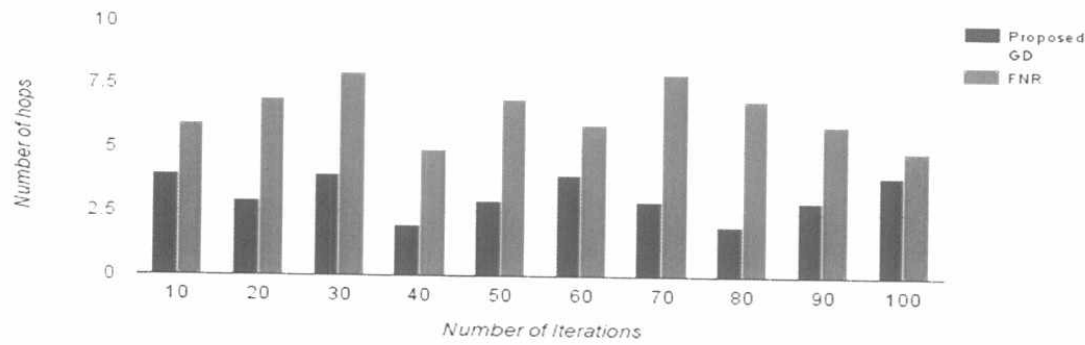

Figure 12 Number of hops of FNR and Proposed GD

The Proposed GD selects the best path whose goal is the minimization of the number of hops between source and sink. In this system, all best paths are saved by keeping power level in focus with different suitable WSN routing schemes converge to this goal faster than the (FNR).

As shown in Figure 13. This result shows the Number of Dead Nodes for the routes discovered using Proposed GD and FNR. 


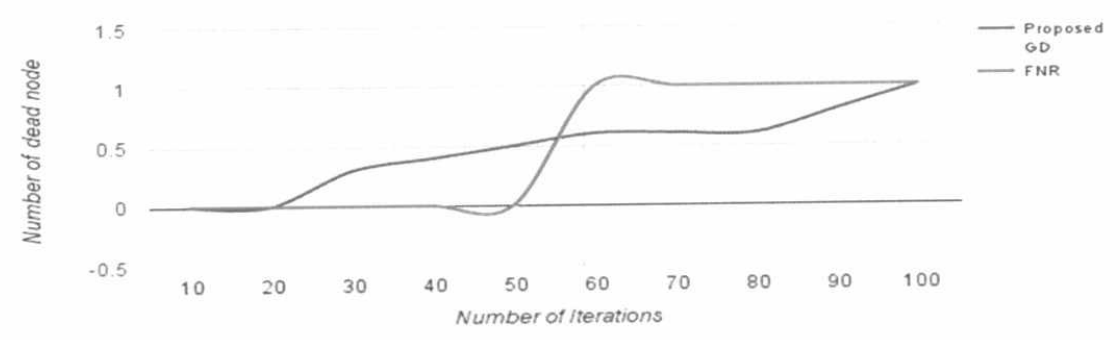

Figure 13. Number of Dead Nodes of FNR and Proposed GD

Implementation will be 50 nodes in the implementation of 100 numbers of iterations that are implemented in the Proposed GD and FNR. The FNR shows result better than Proposed GD when implementing 50 iterations, but after that the proposed system repeats all operations and in result the total number is increased. In case of 60 iterations or more the FNR starts an increase of dead nod but Proposed GD begins to overcome by choosing the sender for all nodes bases the shortest path on the current node power against predefined threshold then determining the amount of power required to perform this operation. After choosing the shortest path, if the power inside the node is enough, it should be selected. If the power inside the node is not enough, it will be isolated and ignored as dead node, which will be replaced by the following node, and then to reconstruct the entire network as it deals with the system in case there are several failed node. The saved information for the Shortest Path deals with the network as a whole.

As shown in Figure 14 result shows the time used to transfer events via using Proposed Grade Diffusion and Fault Node. In this results Proposed Grade Diffusion introduces less time of transfer data from Fault Node then now we tested both systems in same circumstances and below result is discovered.

Time for getting neighbor nodes around faulty nodes per second

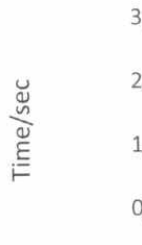

- DFD

Proposed GD

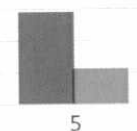

1,45

0,56
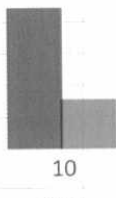

2,22

0,78

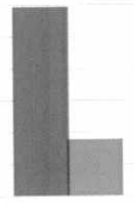

15

2,97

0,89

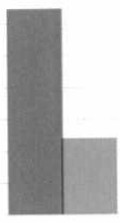

20

3,24

1,2

Figure (14): Time number of neighbor nodes of 2 Algorithms

\section{Conclusions and Future Works}

Wireless sensor is deployed in harsh environments where human intervention is difficult to take place. the biggest issue in designing of WSN is fault node because of the limited power resources of sensors. 
The aim behind the proposed Grade Diffusion method is to keep the sensors working for the longest period possible, thus expanding the lifetime of the network. The power usage of the sensors is overwhelmed by the information transmission and reception. By the Simulation results, the proposed GD reduces the number of hops and dead node and increases the A live nodes compared with FNR. In Future Additional and advanced strategies to improve the performance of the wireless sensor network and make the network more able in detection the fault node and more efficient, has to be desired. Route Determination maintenance strategy also desired to increase the reliability of the data transmissions.

\section{References}

1. R. Mitra and D. Nandy. A survey on clustering techniques for wireless sensor network. Int. J. of Research in Computer Science, 2012.

2. J. L. Hill. System architecture for wireless sensor networks. Ph.D. dissertation, University of California at Berkeley,Spring, 2003.

3. K. Akkaya and M. Younis. A survey on routing protocols for wireless sensor net-works, ad hoc networks. Ad Hoc Networks, 2005.

4. C. Buratti A. Conti D. Dardari and R. Verdone. An overview on wireless sensor networks technology and evolution. Sensors, 2009.

5. N. Chand V. Katiyar and S. Soni. A survey on clustering algorithms for heteroge- neous wireless sensor networks. Int. J. Advanced Networking and Applications, 2011.

6. Anuradha M S1,Deepapatil2 "An Implementation Of Recovery Algorithm For Fault Nodes In A Wireless Sensor Network" International Journal Of Research In Engineering And Technology May-2014.

7. Elmira MoghaddamiKhalilzad, "Misr: Multiple Inheritor Selection For Recovery Of Nodes In Faulty Cluster In Wireless Sensor Networks," Ijcsi International Journal Of Computer Science Issues, Vol. 9, Issue 4, No 1, July 2012.

8. M.Yu, H.Mokhtar, and M.Merabti "A Survey on Fault Management in Wireless Sensor Networks". 2007.

9. Ho, Jiun-Huei, Et Al. "A Ladder Diffusion Algorithm Using Ant Colony Optimization For Wireless Sensor Networks." Information Sciences 192 [2012].

10. Ho, Jiun-Huei, Et Al. "A Ladder Diffusion Algorithm Using Ant Colony Optimization For Wireless Sensor Networks." Information Sciences 192 [2012]. 